\title{
Functional foods for weight management: Dietary Fiber - a systematic review
}

\author{
${ }^{1,2}$ Mona Boaz, ${ }^{3}$ Eyal Leibovitz, and ${ }^{4}$ Julio Wainstein
}

${ }^{1}$ Epidemiology and Research Unit, E. Wolfson Medical Center, Holon, Israel; ${ }^{2}$ Department of Nutrition, School of Health Sciences, Ariel University, Ariel, Israel ; ${ }^{3}$ Internal Medicine Department E, E. Wolfson Medical Center, Holon, Israel; and ${ }^{4}$ Diabetes Unit, E. Wolfson Medical Center, Holon, Israel

Corresponding Author: Prof. Mona Boaz, PhD, Epidemiology and Research Unit, E. Wolfson Medical Center, Halohamim St. 62, Holon 58100, Israel

Submission date: February 12, 2013; Acceptance date: April 29, 2013; Publication date: April 30,2013

\begin{abstract}
It has been estimated that more than 1.5 billion adults are overweight or obese worldwide [1], rendering obesity a global epidemic [2]. Obesity is associated with significant morbidity, including type 2 diabetes, cardiovascular disease, osteoarthritis and some cancers [3]. Thus, obesity is clearly a medical issue, its costs impacting heavily on health care systems in both developed and developing nations [4]. The combined impact of transmissible and chronic disease in the third world is particularly devastating to the very health care systems with fewest resources [5].

Because obesity has been identified as a major health issue, treating obesity is an important goal. However, weight loss management has proven notoriously difficult. It is well documented that reduced energy intake and increased energy expenditure may reduce body weight in the short term, but obesity relapse is the long term is anticipated [6]. In a study of overweight or obese US adults who weighed $\geq 10 \%$ less than their maximum body weight the year prior to the survey $(n=1310), 33.5 \%$ regained $>5 \%$ during that year [7].

Despite its somewhat unimpressive success rate, "lifestyle" weight management remains the first line intervention for obesity treatment [8]. Lifestyle weight management can be defined as interventions based on energy restriction (weight loss diet); increased energy output (exercise); and/or behavioral change (cognitive or behavior therapy). Functional foods have been explored as a tool for enhancing lifestyle weight management.

Functional foods evaluated for their efficacy as obesity interventions can be divided into two broad categories: 1) foods which suppress appetite and increase satiety; and 2) foods which enhance thermogenesis. The present review will focus on those foods thought to act by increasing satiety and suppressing appetite.
\end{abstract}

Key words: Obesity, weight loss, systematic review, dietary fiber 


\section{FOODS WHICH ACT ON APPETITE - DIETARY FIBER}

Dietary fiber can be defined as non-digestible carbohydrates with a degree of polymerization $\geq 3$ [10]. Additionally, a list of beneficial physiological effects of dietary fiber was developed including reduction in blood total and/or LDL cholesterol; attenuation of postprandial blood glucose and/or insulin levels; increased stool bulk and/or decreased transit time; and fermentation by colon microflora. It is noteworthy that additional fiber attributes, such as weight loss/reduced adiposity and increased satiety were not included in the list of beneficial effects [11].

Dietary fiber is not a uniform entity, but rather diverse group of polysaccharides including resistant starch, other glucose polymers with alpha or beta linkages, polymers based on specific sugars such as pectins, fructans and xylans; fiber of marine origin such as alginate and chitosan; and complex fibers rich in pectin, arabinoxylan or beta glucan. Additionally, each of these dietary fibers may be further classified by physiochemical properties including fermentability, solubility and viscosity [12].

One goal of incorporating functional foods into weight management is to enhance satiety and inhibit appetite. Logically, this should reduce food intake and ultimately result in weight loss. Dietary fibers of various types and sources have been proposed as a means through which these weight management goals may be achieved. A systematic review of clinical trials evaluating the impact of dietary fiber interventions on appetite, satiety, energy intake and body weight enables an estimation of its true efficacy.

\section{METHODS}

The association between dietary fiber of any kind and outcomes including satiety, appetite, body weight and/or energy consumption was evaluated by examining clinical trials published during the last two years, from 2010-2012. Search terms included dietary fiber, clinical trial, appetite, and their synonyms. The search was limited by year (2010-2012). Only studies conducted in adults were included.

\section{RESULTS}

A total of 15 studies met the inclusion criteria. Table 1 summarizes the findings. Overall, studies had small sample size and only five (33.3\%) included overweight/obese subjects. Five of the studies measured outcomes 30 min- 8 hours post exposure [13, 14, 19, 20, 23]. Four studies examined outcomes 24 hours past exposure $(15,22,24,27)$, and six studies had longer duration, from 2-15 weeks past exposure [16-18, 21, 25, 26].

Satiety and appetite were evaluated in 10 of the 15 studies $(66.6 \%)$ studies. Of these, $7 / 10$ reported significantly enhanced satiety and $6 / 10$ reported significantly reduced appetite in the fiber intervention compared to the study) control.

Ten of the 15 studies evaluated energy intake. Energy intake was examined in four of the five short term studies, all of which reported a significant reduction in energy intake [13, 14, 19, 23]. All of the 24-hour studies examined energy intake. Two reported reduced dietary intake following fiber exposure [15, 27], one reported no effect on dietary intake [24], and another reported reduced intake in women but increased intake in men [22]. Of the six longer duration studies, two examined energy intake $[17,26]$, neither of which detected a reduction in energy intake by fiber exposure. The association between duration of fiber exposure and proportion of exposed subjects with reduced energy intake is presented in Figure 1. 


\begin{tabular}{|c|c|c|c|c|c|c|c|c|}
\hline Author & Fiber & Control & Subjects & Study Duration & Satiety & Appetite & Energy intake & Body Weight \\
\hline $\begin{array}{l}\text { Smith et al. } \\
2012 \text { (13) }\end{array}$ & Yellow pea fiber & Yellow pea protein & $\begin{array}{l}\text { Healthy males }(\mathrm{n}=19 \\
\text { experiment } 1 ; \mathrm{n}=20 \\
\text { experiment } 2)\end{array}$ & $\begin{array}{l}30 \text { min } \\
\text { (experiment 1); } \\
120 \text { min } \\
\text { (experiment 2) }\end{array}$ & N/A & No effect & $\begin{array}{l}\text { Protein reduced } \\
30 \text { min food } \\
\text { intake vs. fiber; } \\
\text { no effect at } 120 \\
\text { min }\end{array}$ & N/A \\
\hline $\begin{array}{l}\text { Ibrugger et al. } \\
2012 \text { (14) }\end{array}$ & $\begin{array}{l}\text { Flax fiber drink } \\
\text { (exp. 1)or tablets } \\
(\exp 2)\end{array}$ & $\begin{array}{l}\text { Isocaloric/isovolumeric } \\
\text { drink without fiber }\end{array}$ & $\begin{array}{l}\text { Healthy subjects }(\mathrm{n}=24 \text { for } \\
\text { experiment } 1, \mathrm{n}=20 \text { for } \\
\text { experiment } 2)\end{array}$ & $120 \mathrm{~min}$ & $\begin{array}{l}\text { Flax drink } \\
\text { increased satiety } \\
\text { vs. control }\end{array}$ & $\begin{array}{l}\text { Flax drink and } \\
\text { flax tablets } \\
\text { reduced appetite } \\
\text { vs. control }\end{array}$ & $\begin{array}{l}\text { Flax drink } \\
\text { reduced } \\
\text { subsequent } \\
\text { energy intake }\end{array}$ & N/A \\
\hline $\begin{array}{l}\text { Barone et al. } \\
2012(15)\end{array}$ & $\begin{array}{l}\text { B-glucan or } \\
\text { dietary fiber from } \\
\text { fruit }\end{array}$ & Beverage with no fiber & Healthy volunteers $(n=14)$ & 24 hours & $\begin{array}{l}\text { Reduced by } \\
\text { both types of } \\
\text { fiber vs. control }\end{array}$ & $\begin{array}{l}\text { Reduced by } \\
\text { both types of } \\
\text { fiber vs. control }\end{array}$ & $\begin{array}{l}\text { Intake reduced by } \\
\text { B-glucan vs. } \\
\text { control }\end{array}$ & N/A \\
\hline $\begin{array}{l}\text { Jensen et al. } \\
2012 \text { (16) }\end{array}$ & $\begin{array}{l}\text { Low viscous } \\
\text { alginate }\end{array}$ & $\begin{array}{l}\text { Hypo-caloric diet with } \\
\text { placebo supplement }\end{array}$ & Obese subjects $(\mathrm{n}=96)$ & 12 weeks & N/A & N/A & N/A & No effect \\
\hline $\begin{array}{l}\text { Isaksson et al. } \\
2012(17)\end{array}$ & Whole grain rye & Refined wheat & Healthy subjects $(n=24)$ & $\begin{array}{l}\text { Two, 3-week } \\
\text { phases (crossover } \\
\text { design) }\end{array}$ & $\begin{array}{l}\text { Whole grain rye } \\
\text { increased satiety } \\
\text { vs. refined } \\
\text { wheat }\end{array}$ & $\begin{array}{l}\text { Whole grain rye } \\
\text { decreased } \\
\text { appetite vs. } \\
\text { refined wheat }\end{array}$ & No effect & N/A \\
\hline $\begin{array}{l}\text { Jensen et al. } \\
2011 \text { (18) }\end{array}$ & $\begin{array}{l}\text { Low viscous } \\
\text { alginate }\end{array}$ & $\begin{array}{l}\text { Hypo-caloric diet with } \\
\text { no supplement }\end{array}$ & Obese adults $(\mathrm{n}=24)$ & Two weeks & N/A & N/A & N/A & No effect \\
\hline $\begin{array}{l}\text { Calame et al. } \\
2011(19)\end{array}$ & $\begin{array}{l}\text { Two different } \\
\text { blends of gum } \\
\text { arabic dissolved } \\
\text { in water }\end{array}$ & Water & Healthy volunteers $(\mathrm{n}=54)$ & 3 hours & $\begin{array}{l}\text { Both blends } \\
\text { increased satiety } \\
\text { vs. control }\end{array}$ & N/A & $\begin{array}{l}\text { Both blends } \\
\text { reduced intake vs. } \\
\text { control }\end{array}$ & N/A \\
\hline
\end{tabular}




\begin{tabular}{|c|c|c|c|c|c|c|c|c|}
\hline $\begin{array}{l}\text { Isaksson et al. } \\
2011(20)\end{array}$ & $\begin{array}{l}\text { Rye kernels or } \\
\text { mille rye prepared } \\
\text { as bread (exp 1); } \\
\text { or as porridge } \\
(\exp 2)\end{array}$ & Sifted wheat bread & $\begin{array}{l}\text { Healthy volunteers ( } \mathrm{n}=24 \\
\text { for experiment } 1 ; \mathrm{n}=20 \text { for } \\
\text { experiment } 2 \text { ) }\end{array}$ & 8 hours & $\begin{array}{l}\text { Whole and } \\
\text { milled rye } \\
\text { increased satiety } \\
\text { vs. control }\end{array}$ & $\begin{array}{l}\text { Whole and } \\
\text { milled rye } \\
\text { decreased } \\
\text { appetite vs. } \\
\text { control }\end{array}$ & N/A & N/A \\
\hline $\begin{array}{l}\text { Lyon et al. } \\
2011 \text { (21) }\end{array}$ & $\begin{array}{l}\text { High viscosity } \\
\text { polysaccharide }\end{array}$ & Inulin & $\begin{array}{l}\text { Non-dieting overweight or } \\
\text { obese adults ( } n=29 \\
\text { intervention; } n=30 \text { control) }\end{array}$ & 15 weeks & N/A & N/A & N/A & $\begin{array}{l}\text { Weight loss } \\
\text { greater in } \\
\text { intervention } \\
\text { group in } \\
\text { women }\end{array}$ \\
\hline $\begin{array}{l}\text { Hess et al. } \\
2011 \text { (22) }\end{array}$ & $\begin{array}{l}\text { Short chain } \\
\text { fructooligosaccha } \\
\text { rides ( } 5 \text { or } 8 \mathrm{~g} \text { ) as } \\
\text { beverage (dose1) } \\
\text { and } 5 \text { or } 8 \mathrm{~g} \text { as } \\
\text { chew (dose } 2)\end{array}$ & $\begin{array}{l}\text { Beverage not } \\
\text { containing fiber (dose } \\
\text { 1); Chew without fiber } \\
\text { (dose 2) }\end{array}$ & Healthy volunteers $(n=20)$ & 24 hours & $\begin{array}{l}\text { No difference } \\
\text { intervention vs. } \\
\text { control }\end{array}$ & $\begin{array}{l}\text { No difference } \\
\text { intervention vs. } \\
\text { control }\end{array}$ & $\begin{array}{l}\text { No difference } 5 \mathrm{~g} \\
\text { vs. control; } 8 \mathrm{~g} \\
\text { vs. control } \\
\text { reduced intake in } \\
\text { women; increased } \\
\text { intake in men }\end{array}$ & N/A \\
\hline $\begin{array}{l}\text { Monsivais et } \\
\text { al. } 2011 \text { (23) }\end{array}$ & $\begin{array}{l}12 \mathrm{~g} \text { soluble fiber } \\
\text { dextrin or } 11.8 \mathrm{~g} \\
\text { corn fiber or } \\
11.8 \mathrm{~g} \\
\text { polydextrose or } \\
11.2 \mathrm{~g} \text { resistant } \\
\text { starch }\end{array}$ & $\begin{array}{l}\text { Isoenergetic low fiber } \\
\text { control or lower } \\
\text { energy, low fiber } \\
\text { control }\end{array}$ & Healthy adults $(n=36)$ & 4 hours & $\begin{array}{l}\text { All fibers } \\
\text { enhanced satiety } \\
\text { more than low } \\
\text { energy-low fiber } \\
\text { control; no } \\
\text { difference from } \\
\text { isoenergetic-low } \\
\text { fiber control }\end{array}$ & $\begin{array}{l}\text { All fibers } \\
\text { reduced appetite } \\
\text { more than low } \\
\text { energy-low fiber } \\
\text { control; no } \\
\text { difference from } \\
\text { isoenergetic-low } \\
\text { fiber control }\end{array}$ & $\begin{array}{l}\text { Soluble fiber } \\
\text { dextrin } \\
\text { significantly } \\
\text { reduced energy } \\
\text { intake at next } \\
\text { meal vs. } \\
\text { isoenergetic low } \\
\text { fiber control }\end{array}$ & N/A \\
\hline
\end{tabular}




\begin{tabular}{|c|c|c|c|c|c|c|c|c|}
\hline $\begin{array}{l}\text { Juvonen et al. } \\
2011 \text { (24) }\end{array}$ & $\begin{array}{l}10 \mathrm{~g} \text { wheat bran; } \\
10 \mathrm{~g} \text { oat bran; } 5 \mathrm{~g} \\
\text { wheat bran }+5 \mathrm{~g} \\
\text { oat bran presented } \\
\text { in pudding }\end{array}$ & $\begin{array}{l}\text { Pudding with no added } \\
\text { fiber }\end{array}$ & $\begin{array}{l}\text { Normal weight adults } \\
(\mathrm{n}=20)\end{array}$ & 24 hours & $\begin{array}{l}\text { No difference } \\
\text { by exposure }\end{array}$ & $\begin{array}{l}\text { No difference } \\
\text { by exposure }\end{array}$ & $\begin{array}{l}\text { No difference by } \\
\text { exposure }\end{array}$ & N/A \\
\hline $\begin{array}{l}\text { Guerin- } \\
\text { Deremaux et } \\
\text { al. } 2011 \text { (25) }\end{array}$ & $\begin{array}{l}\text { Soluble dextrin } \\
\text { fiber Nutriose in } \\
8,14,18 \text { or } 24 \mathrm{~g} / \mathrm{d} \\
\text { dose in orange } \\
\text { juice }\end{array}$ & $\begin{array}{l}17 \mathrm{~g} \text { maltodextrin in } \\
\text { orange juice }\end{array}$ & $\begin{array}{l}100 \text { overweight healthy } \\
\text { Chinese adults ( } n=20 \text { per } \\
\text { group) }\end{array}$ & 3 weeks & $\begin{array}{l}\text { Immediate } \\
\text { increase in } \\
\text { satiety for } 14, \\
18 \text { and } 24 \mathrm{~g} \\
\text { dose; increased } \\
\text { satiety from day } \\
5 \text { for } 8 \mathrm{~g} \text { dose }\end{array}$ & $\begin{array}{l}\text { Reduced } \\
\text { appetite from } \\
\text { day } 5 \text { for } 24 \mathrm{~g} \\
\text { dose and from } \\
\text { day } 7 \text { for } 14 \text { and } \\
18 \mathrm{~g} \text { dose }\end{array}$ & N/A & N/A \\
\hline $\begin{array}{l}\text { Beck et al. } \\
2010(26)\end{array}$ & $\begin{array}{l}5-6 \mathrm{~g} \text { or } 8-9 \mathrm{~g} \text { oat } \\
\text { beta glucan/day }\end{array}$ & $\begin{array}{l}\text { Energy deficit diet with } \\
\text { no added fiber }\end{array}$ & Overweight women $(\mathrm{n}=66)$ & 3 months & N/A & N/A & $\begin{array}{l}\text { No difference } \\
\text { between groups }\end{array}$ & $\begin{array}{l}\text { No difference } \\
\text { between } \\
\text { groups }\end{array}$ \\
\hline $\begin{array}{l}\text { Bodinham et } \\
\text { al. } 2010(27)\end{array}$ & $\begin{array}{l}48 \mathrm{~g} \text { resistant } \\
\text { starch }+32 \mathrm{~g} \\
\text { rapidly digestible } \\
\text { starch }\end{array}$ & $\begin{array}{l}32 \mathrm{~g} \text { rapidly digestible } \\
\text { starch }\end{array}$ & Healthy males $(n=20)$ & $\begin{array}{l}24 \text { hours on two } \\
\text { separate } \\
\text { occasions }\end{array}$ & $\begin{array}{l}\text { No difference } \\
\text { between groups }\end{array}$ & $\begin{array}{l}\text { No difference } \\
\text { between groups }\end{array}$ & $\begin{array}{l}\text { Resistant starch } \\
\text { significantly } \\
\text { reduced energy } \\
\text { intake }\end{array}$ & N/A \\
\hline
\end{tabular}


Fig. 1 Proportion of subjects with reduced energy intake by duration of fiber intervention

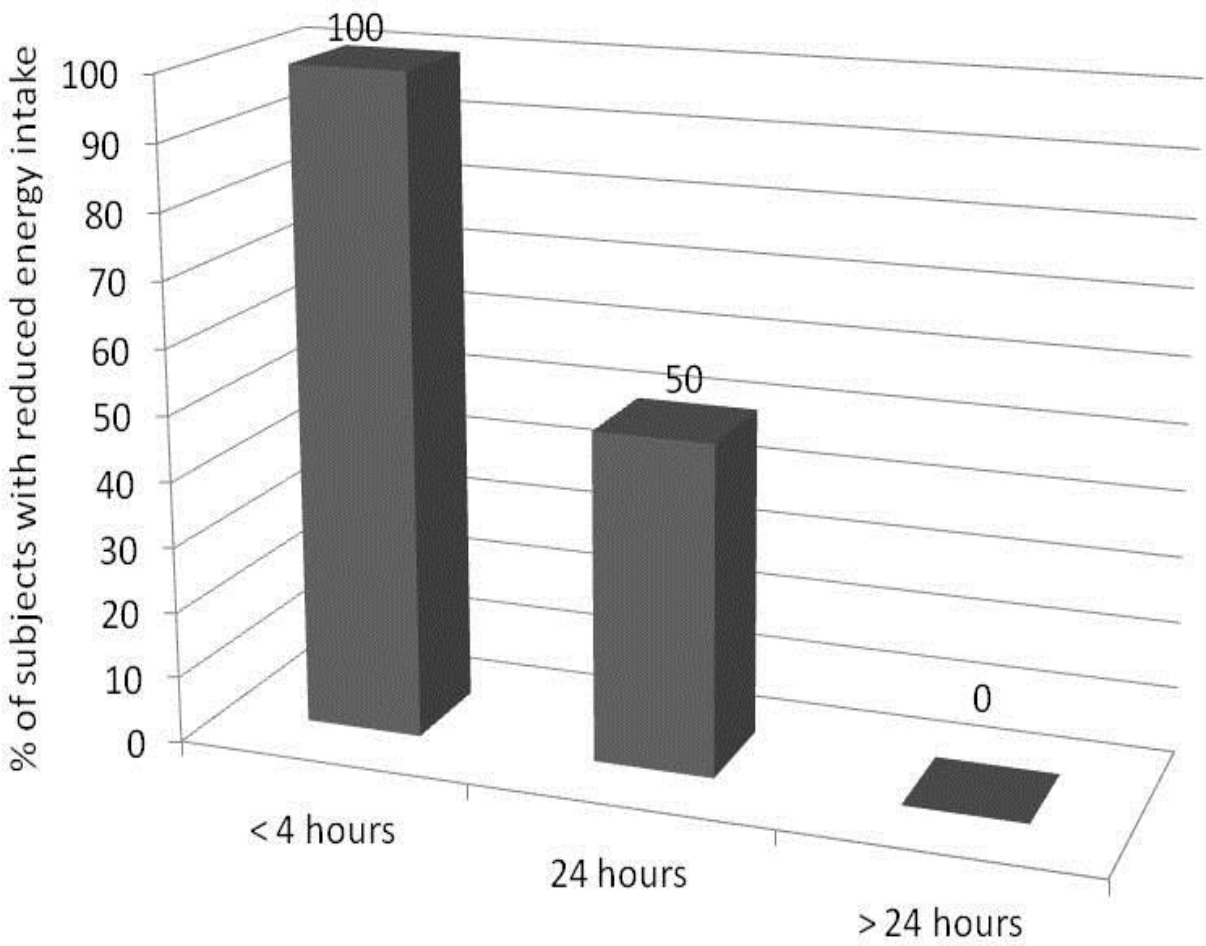

Nine studies examined both satiety/appetite and energy intake [13-15, 17, 19, 22-24, 27], all of which were conducted in healthy, normal weight adults. Four of these studies $[14,15,19,23]$ reported that dietary fiber enhanced satiety/diminished appetite and reduced energy intake; however, one study did not observe a reduction in energy intake despite decreased appetite [17]. Four studies did not observe a difference in satiety/appetite between subjects consuming dietary fiber vs. controls, one of which found no concomitant reduction in energy intake [24]. One study observed reduced energy intake at $30 \mathrm{~min}$ but not $120 \mathrm{~min}$ past fiber exposure [13]. Another study reported no change in energy intake with lower dose fiber $(5 \mathrm{~g})$, but at the higher dose $(8 \mathrm{~g})$, women exhibited reduced energy intake while men increased energy intake [22]. Finally, one study reported reduced energy intake despite no change in satiety/appetite [27].

Body weight was evaluated as an endpoint in four of the 15 studies, all conducted in overweight/obese subjects. Three of these $[16,18,26]$ found that dietary fiber had no effect on body weight compared to placebo, while one study found improved weight loss in women but not in men [21].

\section{DISCUSSION}

Dietary fiber intake is inversely associated with cardiovascular disease, type 2 diabetes, overweight and obesity, metabolic syndrome and its components, as well as gastrointestinal 
diseases [28]. Indeed, viscous dietary fibers have been shown to dampen the post prandial glucose response, reduce total and low density lipoprotein cholesterol, and promote laxation [29].

Mechanisms through which dietary fiber is believed to facilitate weight management include enhancement of satiation and suppression of between-meal appetite [30]. Dietary fiber may influence appetite through the characteristics it imparts to food and also through physiologic effects in the individual. For example, high fiber foods may provide bulk, leading to gastric distention, which creates a sense of satiety [31]. Consistent with this, enhanced satiety and/or reduced appetite were in fact identified in most of the studies measuring this outcome [14, 15, $17,19,20,23,25]$. It is noteworthy that only one of these studies [25] was conducted in overweight patients. All four studies in which dietary fiber was not associated with satiety/appetite were conducted in healthy, normal weight subjects [13, 22, 24, 27].

To contribute to weight management, enhanced satiety/reduced appetite should be associated with reduced energy intake. Nine studies measured both satiety/appetite and energy intake. Five of these reported enhanced satiety/reduced appetite in the fiber group, and four of these five observed concomitant reduction in energy intake [14, 15, 19, 23]. Four studies did not observe satiety enhancement/appetite reduction, and not surprisingly, decreased energy intake was not recorded $[13,22,24]$. Interestingly, one study detected decreased energy intake at the post exposure test meal and for the next 24 hours despite no change in satiety/appetite [27]. The 24-hour energy reduction was driven by reduced fat intake, though no change in desire for fatty foods was indicated by participants on questionnaires.

\section{CONCLUSIONS}

Dietary fiber appears to enhance satiety and reduce appetite, but this is not consistently associated with reduced energy intake, particularly in the long term. Most of the studies evaluated were conducted in normal weight individuals. There is a need for well-designed randomized, controlled clinical trials in overweight and/or obese individuals in order to assess the efficacy of dietary fiber as a weight loss strategy.

\section{Competing Interests}

The present study received no funding and all authors declare that they have no competing interests.

\section{Author Contributions}

MB conceived of the study, reviewed articles for inclusion, conducted the analyses and wrote the manuscript. EL and JW reviewed articles for inclusion, edited the manuscript and approved the final version of the paper.

\section{REFERENCES}

1. Nguyen T, Lau DC. The obesity epidemic and its impact on hypertension. Can J Cardiol 2012; 28:326-33. 
2. Ginter E, Simko V. Type 2 diabetes mellitus, pandemic in $21^{\text {st }}$ century. Adv Exp Med Biol 2012; 771:42-50.

3. Pi-Sunyer X. The medical risks of obesity. Postgrad Med 2009; 121: 21-33.

4. Magnusson RS. Rethinking global health challenges: towards a 'global compact' for reducing the burden of chronic disease. Public Health 2009; 123:265-74.

5. Borch-Johnsen K. The metabolic syndrome in a global perspective. The public health impact--secondary publication. Dan Med Bull 2007;

6. Kissler HJ, Settmacher U. Bariatric surgery to treat obesity. Semin Nephrol 2013; 33: 75-89.

7. Sumithran P, Proietto J. The defence of body weight: a physiological basis for weight regain after weight loss. Clin Sci (Lond) 2013; 124:231-41.

8. Weiss EC, Galuska DA, Kettel Khan L, Gillespie C, Serdula MK. Weight regain in U.S. adults who experienced substantial weight loss, 1999-2002. Am J Prev Med 2007; 33:34-40.

9. Hainer V, Hainerová IA. Do we need anti-obesity drugs? Diabetes Metab Res Rev 2012; 28 Suppl 2:8-20.

10. Jones JM. Dietary fiber future directions: integrating new definitions and findings to inform nutrition research and communication. Adv Nutr 2013; 4:8-15.

11. Howlett JF, Betteridge VA, Champ M, Craig SA, Meheust A, Jones JM. The definition of dietary fiber - discussions at the Ninth Vahouny Fiber Symposium: building scientific agreement. Food Nutr Res. 2010 Nov 1;54.

12. Wanders AJ, van den Borne JJGC, de Graaf C, Hulshof T, Jonathan MC, Kristensen M, Mars M, Schols HA, Feskens EJM. Effects of dietary fibre on subjective appetite, energy intake and body weight: a systematic review of randomized controlled trials. Obesity Rev 2011; 12: 724-39.

13. Smith CE, Mollard RC, Luhovyy BL, Anderson GH. The effect of yellow pea protein and fibre on short-term food intake, subjective appetite and glycaemic response in healthy young men. Br J Nutr 2012;108 Suppl 1: S74-80.

14. Ibrügger S, Kristensen M, Mikkelsen MS, Astrup A. Flaxseed dietary fiber supplements for suppression of appetite and food intake. Appetite. 2012; 58: 490-5.

15. Barone Lumaga R, Azzali D, Fogliano V, Scalfi L, Vitaglione P. Sugar and dietary fibre composition influence, by different hormonal response, the satiating capacity of a fruit-based and a $\beta$-glucan-enriched beverage. Food Funct 2012; 3: 67-75.

16. Georg Jensen M, Kristensen M, Astrup A. Effect of alginate supplementation on weight loss in obese subjects completing a 12-wk energy-restricted diet: a randomized controlled trial. Am J Clin Nutr 2012; 96:5-13.

17. Isaksson Isaksson H, Tillander I, Andersson R, Olsson J, Fredriksson H, Webb DL, Åman P. Whole grain rye breakfast - sustained satiety during three weeks of regular consumption. Physiol Behav 2012; 105: 877-84.

18. Jensen GM, Kristensen M, Astrup A. Can alginate-based preloads increase weight loss beyond calorie restriction? A pilot study in obese individuals. Appetite 2011; 57: 6014. 
19. Calame W, Thomassen F, Hull S, Viebke C, Siemensma AD. Evaluation of satiety enhancement, including compensation, by blends of gum arabic. A methodological approach. Appetite 2011; 57: 358-64.

20. Isaksson H, Rakha A, Andersson R, Fredriksson H, Olsson J, Aman P. Rye kernel breakfast increases satiety in the afternoon - an effect of food structure. Nutr J 2011; 10:31-41.

21. Lyon M, Wood S, Pelletier X, Donazzolo Y, Gahler R, Bellisle F. Effects of a 3-month supplementation with a novel soluble highly viscous polysaccharide on anthropometry and blood lipids in nondieting overweight or obese adults. J Hum Nutr Diet 2011; 24: 351-9.

22. Hess JR, Birkett AM, Thomas W, Slavin JL. Effects of short-chain fructooligosaccharides on satiety responses in healthy men and women. Appetite 2011; 56: $128-34$.

23. Monsivais P, Carter BE, Christiansen M, Perrigue MM, Drewnowski A. Soluble fiber dextrin enhances the satiating power of beverages. Appetite 2011; 56:9-14.

24. Juvonen KR, Salmenkallio-Marttila M, Lyly M, Liukkonen KH, Lähteenmäki L, Laaksonen DE, Uusitupa MI, Herzig KH, Poutanen KS, Karhunen LJ. Semisolid meal enriched in oat bran decreases plasma glucose and insulin levels, but does not change gastrointestinal peptide responses or short-term appetite in healthy subjects. Nutr Metab Cardiovasc Dis 2011; 21: 748-56.

25. Guérin-Deremaux L, Pochat M, Reifer C, Wils D, Cho S, Miller LE. The soluble fiber NUTRIOSE induces a dose-dependent beneficial impact on satiety over time in humans. Nutr Res 2011; 31:665-72.

26. Beck EJ, Tapsell LC, Batterham MJ, Tosh SM, Huang XF. Oat beta-glucan supplementation does not enhance the effectiveness of an energy-restricted diet in overweight women. Br J Nutr 2010; 103:1212-22.

27. Bodinham CL, Frost GS, Robertson MD. Acute ingestion of resistant starch reduces food intake in healthy adults. Br J Nutr 2010; 103:917-22.

28. Timm D, Slavin J. Dietary fiber and the relationship to chronic diseases. Am J Lifestyle Med 2008; 2: 233-40.

29. Chutkan R, Fahey G, Wright WL, McRorie J. Viscous versus nonviscous soluble fiber supplements: mechanisms and evidence for fiber-specific health benefits. J Am Acac Nurse Pract 2012; 24: 476-87.

30. Halford JCG, Boyland EJ, Blundell JE, Kirkham TC, Harrold JA. Pharmacological management of appetite expression in obesity. Nat Rev Endocrinol 2010; 6: 255-69.

31. Dikerman C, Fahey G. Viscosity as related to dietary fiber: a review. Crit Rev Food Sci Nutr 2006; 46: 649-63. 